\title{
HIGHLIGHTS
}

BIOMARKERS

\section{Elevated microparticle levels in primary Sjögren's syndrome}

Increased levels of microparticlesphosphatidylserine-rich vesicles shed from the plasma membranes of activated cells-in patients with primary Sjögren's syndrome (pSS), systemic lupus erythematosus (SLE) and rheumatoid arthritis (RA) highlight their potential as a biomarker in autoimmune disease. "Increased plasma levels of microparticles have been reported in autoimmune disease but we report the first assessment of circulating microparticle levels in pSS," write Sellam et al. in their recently published Arthritis Research \& Therapy paper.

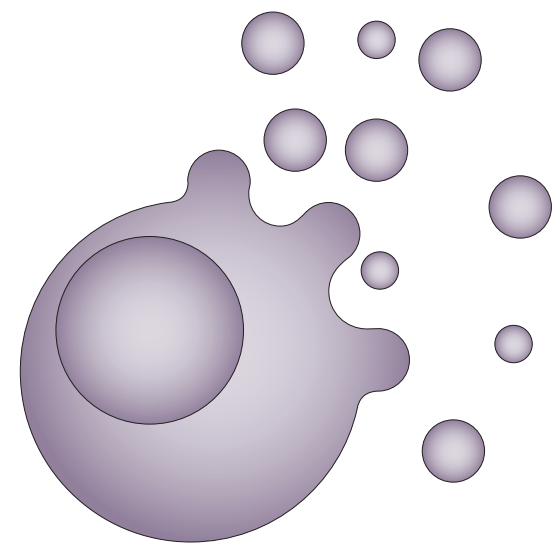

The researchers measured levels of total, platelet and leukocyte microparticles in 43 patients with pSS, 20 with SLE, 24 with RA and 44 healthy individuals. All patients had considerably increased plasma concentrations of total and platelet microparticles in comparison with healthy controls. Only patients with pSS had significantly elevated levels of leukocyte microparticles $(P<0.0001)$. In patients with pSS, however, platelet microparticle concentration was significantly lower in individuals with severe disease (extraglandular involvement) than in those with only glandular involvement $(P=0.02)$. Furthermore, platelet microparticle levels inversely correlated with those of serum $\beta 2$ microglobulin and anti-doublestranded DNA IgG in participants with pSS and SLE, respectively, while total microparticle levels inversely correlated with secretory phospholipase A2-an enzyme that degrades phospholipids-in all patients.

The investigators suggest that increased microparticle levels in autoimmune disease could be used as a biomarker that reflects systemic cell activation, although they would be of limited use in assessing

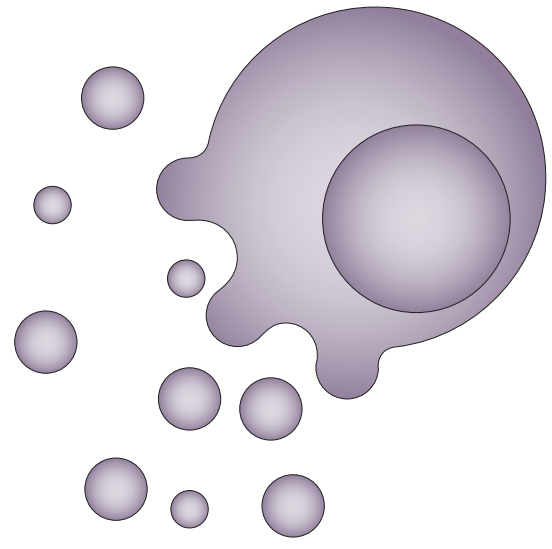

symptoms in patients with chronic disease. In addition, the authors suggest that platelet activation might have a role in the pathogenesis of pSS. "The role of platelets in pSS immunopathology should now be investigated, especially their involvement in the interferon signature found in pSS," concludes Xavier Mariette, the study's lead investigator.

Rowan Higgs

Original article Sellam, J. et al. Increased levels of circulating microparticles in primary Sjögren's syndrome, systemic lupus erythematosus and rheumatoid arthritis and relation with disease activity. Arthritis Res. Ther. 11, R156 (2009)

Further reading Beyer, C. \& Pisetsky, D. The role of microparticles in the pathogenesis of rheumatic diseases. Nat. Rev. Rheumatol. 6, 21-29 (2010) 